\title{
Fourier Interpolation and Time-Frequency Localization
}

\author{
Aleksei Kulikov ${ }^{1}$
}

Received: 31 August 2020 / Revised: 11 May 2021 / Accepted: 25 May 2021 /

Published online: 10 June 2021

(c) The Author(s) 2021

\section{Abstract}

We prove that under very mild conditions for any interpolation formula $f(x)=$ $\sum_{\lambda \in \Lambda} f(\lambda) a_{\lambda}(x)+\sum_{\mu \in M} \hat{f}(\mu) b_{\mu}(x)$ we have a lower bound for the counting functions $n_{\Lambda}\left(R_{1}\right)+n_{M}\left(R_{2}\right) \geq 4 R_{1} R_{2}-C \log ^{2}\left(4 R_{1} R_{2}\right)$ which very closely matches recent interpolation formulas of Radchenko and Viazovska and of Bondarenko, Radchenko and Seip.

Keywords Fourier interpolation - Local cosine bases $\cdot$ Prolate spheroidal wave functions

Mathematics Subject Classification Primary 42A16; Secondary 42A20 • 42C05

\section{Introduction}

In the recent breakthrough paper [7] Radchenko and Viazovska showed that any Schwartz function can be effectively reconstructed from the values of it and its Fourier transform at the points $\pm \sqrt{n}, n \in \mathbb{Z}_{\geq 0}$ and two more values $f^{\prime}(0), \hat{f}^{\prime}(0)$. If we consider the counting function $n_{\Lambda}(R)=|\Lambda \cap[-R, R]|$, which in the case $\Lambda=\{ \pm \sqrt{n}\}$ takes the form $n_{\Lambda}(R)=1+2\left[R^{2}\right]$, we see that it satisfies the inequality $n_{\Lambda}(W)+n_{\Lambda}(T) \geq 4 W T-O(1)$ for all $W, T$. We observe that this bound perfectly matches the famous $4 W T$ Theorem of Slepian [10] which says that the space of functions which are supported on $[-T, T]$ and such that their Fourier transforms are

Communicated by Hans G. Feichtinger.

This work was supported by Grant 275113 of the Research Council of Norway and in part by the Moebius Contest Foundation for Young Scientists.

$凶$ Aleksei Kulikov

lyosha.kulikov@mail.ru

1 Department of Mathematical Sciences, Norwegian University of Science and Technology, 7491

Trondheim, NO, Norway 
essentially supported on $[-W, W]$ has approximate dimension $4 W T .{ }^{1}$ We prove that this is not a coincidence and that a similar inequality holds for all such interpolation formulas with a very small error term.

Theorem 1.1 Let $\Lambda, M \subset \mathbb{R}$ be two multisets and $L$ be some fixed number. Assume that the following interpolation formula holds for all $C^{L}$ compactly supported functions $f$

$$
f(x)=\sum_{\lambda \in \Lambda} f^{(r(\lambda))}(\lambda) a_{\lambda}(x)+\sum_{\mu \in M} \hat{f}^{(k(\mu))}(\mu) b_{\mu}(x),
$$

where $r: \Lambda \rightarrow \mathbb{Z}_{\geq 0}, k: M \rightarrow \mathbb{Z}_{\geq 0}$ and $a_{\lambda}, b_{\mu}: \mathbb{R} \rightarrow \mathbb{C}, \lambda \in \Lambda, \mu \in M$. Assume additionally that $r$ and $k$ are at most $L$, the counting function of $M$ satisfies the bound $n_{M}(R) \leq R^{L}$ for large enough $R$ and that $b_{\mu}(x)$ is polynomially bounded in $\mu$ and $x$. Then there exists $C>0$ depending only on the interpolation formula above such that for all $R_{1}, R_{2}>1$

$$
n_{\Lambda}\left(R_{1}\right)+n_{M}\left(R_{2}\right) \geq 4 R_{1} R_{2}-C \log ^{2}\left(4 R_{1} R_{2}\right) .
$$

Remark 1.2 Note that since the right-hand side of (1.1) is continuous in the space of compactly supported $C^{K}$ functions for big enough $K$, by the density argument it is enough to assume that the formula holds only for the $C^{\infty}$ compactly supported functions, which is a natural space for which the right-hand side of (1.1) is defined.

This result reflects the idea that for a function satisfying the conditions of Slepian's theorem, the values of this function and its Fourier transform outside of the corresponding intervals are mostly irrelevant and to generate an $N$-dimensional vector space we need at least $N$ vectors (although the "space" under consideration is by no means a vector space).

A way to make the $4 W T$ Theorem of Slepian precise is to consider the so-called prolate spheroidal wave functions, studied by Slepian, Landau and Pollak $[5,9,11]$. These functions are the eigenvectors of the time-frequency localization operator corresponding to the intervals $[-W, W]$ and $[-T, T]$. Recently, there has been a revival of interest in these functions and especially in the distribution of the corresponding eigenvalues, see e.g. [3]. The key ingredient in our proof is a recent sharp estimate for these eigenvalues proved in [4].

Let us emphasize that for the conclusion of our theorem to hold, it is not enough to assume only uniqueness i.e. that any function which vanishes on $\Lambda$ and whose Fourier transform vanishes on $M$ is zero. We need some quantitative assumption like an interpolation formula or frame property. To illustrate this, let us mention a wellknown result of Ascensi, Lyubarskii and Seip [1], which gives us a uniqueness result (but not an interpolation formula) with effectively half the number of points.

Theorem 1.3 (Ascensi, Lyubarskii, Seip) Let $f \in L^{2}(\mathbb{R})$. Assume that it is orthogonal to the functions $\exp \left(-\pi(t+\lambda)^{2}\right), \lambda \in \Lambda$ and $\exp \left(-\pi t^{2}+2 \pi i \mu t\right), \mu \in M$ with $\Lambda=\{ \pm \sqrt{2 n}\}$ and $M=\{ \pm \sqrt{2 n}\} \cup\{-1,1\}$. Then this function is identically zero.

\footnotetext{
1 Slepian called it the $2 W T$ Theorem since he considered intervals $[-W, W]$ and $[-T / 2, T / 2]$ which is more natural from the engineering point of view.
} 
If we think of a Gaussian as a smoothed version of the $\delta$-function used in formula (1.1) then this result gives us a uniqueness set with half as many points as claimed in the Theorem 1.1. Nevertheless there is no contradiction between Theorem 1.1 and Theorem 1.3 because because it is impossible to reconstruct a function $f$ in a stable way from its scalar products with the functions from the Theorem 1.3.

The usual way to express effective reconstruction is by imposing the frame property. Recall that the set of vectors $\left\{v_{k}\right\}$ in the Hilbert space $\mathcal{H}$ is said to be a frame if for all $f \in \mathcal{H}$ we have $\|f\|^{2} \sim \sum_{k}\left|\left\langle f, v_{k}\right\rangle\right|^{2}$. In this language we can say that the set of functions from Theorem 1.3 is extremely far from being a frame (even if we only care about a lower bound for $\|f\|^{2}$ and put a fairly large weight on $\left|\left\langle f, v_{k}\right\rangle\right|^{2}$ ), which can be deduced from the (proof of a) general result of Seip [8] or seen directly by considering the functions $f_{N}(t)=\exp \left(-\pi(t+N)^{2}+2 \pi i N t\right), N \rightarrow \infty$. Theorem 1.1 shows that a density condition akin to that of [8] holds under a much weaker assumption about reconstruction than what follows from the frame property.

Let us also mention a recent interpolation formula of Bondarenko, Radchenko and Seip [2]. They proved that, under suitable conditions, one can recover the value $\hat{f}(x)$ by means of an interpolation formula from the values of $f$ at the points $\frac{ \pm \log (n)}{4 \pi}$ and the values of $\hat{f}$ at the points $\frac{\rho-1 / 2}{i}$ with $\rho$ ranging over the nontrivial zeros of the Riemann zeta function. Although in the absence of the Riemann hypothesis these points can be non-real and the formula from [2] converges only after some grouping of terms, one can still apply our techniques to their setting and get the bound

$$
2 N(T)+2 e^{4 \pi W} \geq 4 W T-C \log ^{2}(W T),
$$

where $N(T)$ is the number of zeros $\rho$ of the Riemann zeta function with $0<\Im(\rho)<T$. Choosing $W=\frac{1}{4 \pi} \log \left(\frac{T}{2 \pi}\right)$ we get the lower bound which matches the Riemann-von Mangoldt formula up to the power of the logarithmic term

$$
N(T) \geq \frac{T}{2 \pi} \log \left(\frac{T}{2 \pi e}\right)-C \log ^{2}(T) .
$$

Finally let us remark that our result admits a natural generalization to the space of even/odd functions with $4 W T$ replaced by $2 W T$. This result also perfectly matches interpolation formulas from [2] and [7].

\section{Prolate Spheroidal Wave Functions}

In this section we will formulate the basic properties of the prolate spheroidal wave functions that we need in our proof, as well as the bound for the corresponding eigenvalues from [4] which is the main nontrivial result that we use.

For the intervals $I=\left[-\frac{1}{2}, \frac{1}{2}\right], J=\left[-\frac{c}{2} \cdot \frac{c}{2}\right]$ we define an operator $T_{I, J}=$ $P_{I} \mathcal{F}^{-1} P_{J} F: L^{2}(I) \rightarrow L^{2}(I)$, where $\mathcal{F}$ is the Fourier transform $\mathcal{F} f(\xi)=\hat{f}(\xi)=$ $\int_{\mathbb{R}} f(x) e^{-2 \pi i x \xi} d x$ and $P_{I}, P_{J}$ are the projections from $L^{2}(\mathbb{R})$ to $L^{2}(I)$ and $L^{2}(J)$ respectively. $T_{I, J}$ is a self-adjoint compact positive operator and as such it has a 
complete set of eigenvectors $\varphi_{n, c}$ and corresponding eigenvalues $\lambda_{n}(c)$. The prolate spheroidal wave functions $\psi_{n, c}(\xi)$ are defined as the Fourier transforms of $\varphi_{n, c}(x)$ normalized so that $\left\|\psi_{n, c}\right\|_{L^{2}(\mathbb{R})}=1$.

Let us list the basic properties of these functions which can be found in e.g. [11]

(i) $\psi_{n, c}$ are entire functions,

(ii) $\psi_{n, c}$ and $\psi_{m, c}$ are orthogonal for $n \neq m$,

(iii) $\operatorname{supp} \hat{\psi}_{n, c}=\left[-\frac{1}{2}, \frac{1}{2}\right]$,

(iv) $\lambda_{n}(c)=\left\|\psi_{n, c}\right\|_{L^{2}([-c / 2, c / 2])}$,

(v) $\hat{\psi}_{n, c}(x)=\alpha_{n}(c) \psi_{n, c}(c x),|x|<\frac{1}{2}$, where $\alpha_{n}(c)=\frac{\sqrt{c}}{\lambda_{n}(c)}$.

(vi) $\psi_{n, c}$ is even if $n$ is even and odd if $n$ is odd.

The key nontrivial result about the prolate spheroidal wave functions is the following theorem which is Corollary 3 from [4].

Theorem 2.1 For any $c>0$ and $n<c-2$ we have

$$
\lambda_{n}(c) \geq 1-10 \exp \left(\frac{n-c-6}{\frac{2}{\pi^{2}} \log (50 c+25)}\right) .
$$

Note that this statement is a bit different from Corollary 3 from [4] because we use a different normalization of the Fourier transform.

We will now deduce some corollaries from this theorem which are easier to use.

Corollary 2.2 For every fixed number $A>1$ there exists a constant $B>1$ such that for $c>4$ and $n<c-B \log ^{2}(c)$ we have

$$
\lambda_{n}(c) \geq 1-\frac{10}{c^{A}}
$$

Proof If $n<c-B \log ^{2}(c)$ and $c>4$ then under the exponent in the formula (2.1) we have at most

$$
-\frac{B \log (c)^{2}}{1000 \log (c)}=-\frac{B}{1000} \log (c) .
$$

Therefore choosing $B=1000 A$ we get the result.

To pass from the bound for the eigenvalues to a pointwise bound we need the following lemma the proof of which we postpone to the Section 4.

Lemma 2.3 For every $k \in \mathbb{N}_{0}$ there exists a constant $C_{k}$ such that for all $f \in L^{2}(\mathbb{R})$ with supp $\hat{f} \subset\left[-\frac{1}{2}, \frac{1}{2}\right]$ we have

$$
\left|f^{(k)}(x)\right| \leq C_{k}\|f\|_{L^{2}(\mathbb{R})}^{1 / 2}\|f\|_{L^{2}([x,+\infty))}^{1 / 2} .
$$

From this lemma and Corollary 2.2 we get the following. 
Corollary 2.4 For any number $D>0$ and $k \in \mathbb{N}_{0}$ there exists constants $B$ and $C$ such that for $c>4, n<c-B \log ^{2}(c)$ and $\xi$ with $|\xi|>\frac{c}{2}$ we have

$$
\left|\psi_{n, c}^{(k)}(\xi)\right| \leq \frac{C}{c^{D}} .
$$

Proof Without loss of generality we can assume that $\xi>\frac{c}{2}$. Since supp $\hat{\psi}_{n, c} \subset$ $\left[-\frac{1}{2}, \frac{1}{2}\right]$ we have

$$
\begin{aligned}
\left|\psi_{n, c}^{(k)}(\xi)\right| & \leq C_{k}\left\|\psi_{n, c}\right\|_{L^{2}(\mathbb{R})}^{1 / 2}\left\|\psi_{n, c}\right\|_{L^{2}([\xi,+\infty))}^{1 / 2} \leq C_{k}\left\|\psi_{n, c}\right\|_{L^{2}([c / 2,+\infty))}^{1 / 2} \\
& \leq C_{k}\left(1-\lambda_{n}(c)^{2}\right)^{1 / 4}
\end{aligned}
$$

The bound (2.4) now follows from (2.2) if $A$ is big enough depending on $D$.

A final thing that we need is the following crude bound for the derivatives of the functions $\hat{\psi}_{n, c}$ on the interval $\left(-\frac{1}{2}, \frac{1}{2}\right)$.

Proposition 2.5 For every $m \geq 0$ there exists constants $A_{m}, B_{m}$ such that for $c>4$ and $n<c-100 \log (c)$ we have

$$
\left|\hat{\psi}_{n, c}^{(m)}(x)\right| \leq A_{m} c^{B_{m}},|x|<\frac{1}{2} .
$$

Proof First of all we note that if $c>4$ and $n<c-100 \log (c)$ then the inequality (2.1) implies that $\lambda_{n}(c) \geq \frac{1}{2}$ (note that we can get a similar bound using the classical result of Landau and Widom [6]). Therefore, in the fifth property of the prolate spheroidal wave functions we have $\alpha_{n}(c) \leq 2 \sqrt{c}$ and so proving bounds for the derivatives of $\hat{\psi}_{n, c}$ is the same as proving bounds for the derivatives of $\psi_{n, c}$. To do so we again use Lemma 2.3 and get

$$
\left|\psi_{n, c}^{(m)}(x)\right| \leq C_{m}\left\|\psi_{n, c}\right\|_{L^{2}(\mathbb{R})}^{1 / 2}\left\|\psi_{n, c}\right\|_{L^{2}([x,+\infty))}^{1 / 2} \leq C_{m}\left\|\psi_{n, c}\right\|_{L^{2}(\mathbb{R})}=C_{m}
$$

which, after a change of variables, transforms into the bound (2.6).

\section{Proof of Theorem 1.1}

Put $c=4 R_{1} R_{2}$ and assume that $n_{\Lambda}\left(R_{1}\right)+n_{M}\left(R_{2}\right)<c-C \log ^{2}(c)$. We also fix a big enough integer $K$ to be determined later. Consider the function $f$ with $\hat{f}(x)=$ $\sum_{n=0}^{N} a_{j} \psi_{n, c}\left(2 R_{1} x\right)$ for some numbers $a_{j} \in \mathbb{C}$, where $N=n_{\Lambda}\left(R_{1}\right)+n_{M}\left(R_{2}\right)+$ $2 K+2$. Note that $f$ is a restriction of an entire function to the interval $\left(-R_{1}, R_{1}\right)$ so we can speak of it and its derivatives at the points $\pm R_{1}$ understood as the limits from the appropriate sides. By the linear algebra argument we can choose nontrivial $a_{j}$ so that $f^{(r(\lambda))}(\lambda)=0,|\lambda| \leq R_{1}, \hat{f}^{(k(\mu))}(\mu)=0,|\mu| \leq R_{2}$ and $f\left( \pm R_{1}\right)=f^{\prime}\left( \pm R_{1}\right)=$ $\ldots=f^{(K)}\left( \pm R_{1}\right)=0$. This in particular implies that $f \in C^{K}(\mathbb{R})$. For convenience 
we normalize $a_{n}$ so that $\sum\left|a_{n}\right|^{2}=1$. Finally, we assume for now that the number $c$ is big enough.

Let us write the interpolation formula (1.1) for the function $f$ (it is valid as long as $K \geq L)$

$$
f(x)=\sum_{\lambda \in \Lambda} f^{(r(\lambda))}(\lambda) a_{\lambda}(x)+\sum_{\mu \in M} \hat{f}^{(k(\mu))}(\mu) b_{\mu}(x) .
$$

Note that by assumption $f^{(r(\lambda))}(\lambda)=0,|\lambda| \leq R_{1}$ and $\hat{f}^{(k(\mu))}(\mu)=0,|\mu| \leq R_{2}$. Moreover, since the function $f$ is supported on $\left[-R_{1}, R_{1}\right]$, we have $f^{(r(\lambda))}(\lambda)=$ $0,|\lambda|>R_{1}$. Therefore, formula (3.1) simplifies to

$$
f(x)=\sum_{\mu \in M,|\mu|>R_{2}} \hat{f}^{(k(\mu))}(\mu) b_{\mu}(x) .
$$

We are going to prove two estimates for $\hat{f}^{(s)}(x),|x|>R_{2}$ with fixed $s$, one of which will be useful for small values of $x$ and the other one for big values of $x$. We begin with the first one.

If $c$ is big enough then we have $c-C \log ^{2}(c)+2 K+2<c-\frac{C}{2} \log ^{2}(c)$. For any fixed $T>1$ if $\frac{C}{2}$ is big enough then from the bound (2.4) we have for any $x$ with $|x|>\frac{c}{2}$

$$
\left|\psi_{n, c}^{(s)}(x)\right| \leq \frac{C_{s, T}}{c^{T}}, 0 \leq n \leq N
$$

Since $\sum\left|a_{n}\right|^{2}=1$ we obviously have $\left|a_{n}\right| \leq 1$ for each $n$. Also if $c$ is big enough then $N<c$. Therefore by summing the estimates (3.3) over all $n$ we get

$$
\left|\hat{f}^{(s)}(x)\right| \leq \frac{C_{s, T}}{c^{T-1}},|x|>R_{2} .
$$

This bound is good for not too big $x$ but since the right-hand side does not depend on $x$ it is not enough by itself. For large values of $x$ we will use that $f \in C^{K}(\mathbb{R})$ and integrate by parts $K$ times. We have

$$
\mathcal{F}\left(\left((2 \pi i y)^{s} f(y)\right)^{(K)}\right)(x)=(2 \pi i x)^{K} \hat{f}^{(s)}(x) .
$$

Since $|y| \leq R_{1} \leq c$ using the bound (2.6) and the trivial estimate $|\mathcal{F}(g)(x)| \leq$ $\|g\|_{L^{1}(\mathbb{R})}$ we get that there exist constants $U_{K, s}, V_{K, s}$ such that

$$
\left|\hat{f}^{(s)}(x)\right| \leq \frac{U_{K, s} c^{V_{K, s}}}{|x|^{K}}
$$

Taking the geometric mean of the bounds (3.4) and (3.6) we get for $|x|>R_{2}$

$$
\left|\hat{f}^{(s)}(x)\right| \leq \sqrt{C_{s, T} U_{K, s}} c^{V_{K, s} / 2-T / 2+1 / 2}|x|^{-K / 2} .
$$


Choosing $K$ big enough and then $T$ big enough depending on $K$ (that is, $C$ big enough since $T$ depends on $C$ ) we get for all $s=0, \ldots, L$

$$
\left|\hat{f}^{(s)}(x)\right| \leq A_{s, K} c^{-K / 2}|x|^{-K / 2} .
$$

Plugging this bound into formula (3.2) and recalling that we assume polynomial bounds for $b_{\mu}(x)$ and the counting function of the set $M$ we see that for $K$ big enough we can get

$$
|f(x)| \leq \frac{F}{c^{2}},|x|<R_{1}
$$

for some absolute constant $F$. On the other hand, since $\|f\|_{L^{2}(\mathbb{R})}=\frac{1}{\sqrt{2 R_{1}}}$ and supp $f=\left(-R_{1}, R_{1}\right)$, there exists $x \in\left(-R_{1}, R_{1}\right)$ such that $|f(x)| \geq \frac{1}{2 R_{1}} \geq \frac{1}{c}$. This gives us a contradiction for big enough $c$. To prove the theorem for small $c$ as well we artificially enlarge $C$ so that $c-C \log ^{2}(c)$ is negative and there's nothing to prove.

Finally, if the interpolation formula is true only for even/odd functions $f$ then the same argument with using only even/odd prolate spheroidal wave functions gives us a bound $2 R_{1} R_{2}-C \log ^{2}\left(2 R_{1} R_{2}\right)$.

\section{Proof of Lemma 2.3}

To prove Lemma 2.3 we need the following purely real-variable lemma.

Lemma 4.1 For all $k, n \in \mathbb{N}_{0}, k<n$ there exists $C_{k, n}>0$ such that for all $f \in$ $C^{n}([0,+\infty)) \cap L^{2}([0,+\infty))$ we have for $\alpha_{k, n}=\frac{n-k-1 / 2}{n}$

$$
\left|f^{(k)}(0)\right| \leq C_{k, n}\|f\|_{L^{2}([0,+\infty))}^{\alpha_{k, n}}\left\|f^{(n)}\right\|_{L^{2}([0,+\infty))}^{1-\alpha_{k, n}}
$$

Proof By replacing the function $f$ with the function $g(x)=a f(b x)$ we can without loss of generality assume that $\|f\|_{L^{2}([0,+\infty))}=\left\|f^{(n)}\right\|_{L^{2}([0,+\infty))}=1$ (note that at this step we use the exact value of $\alpha_{k, n}$ so that the left-hand side and the right-hand side of (4.1) are multiplied by the same number after this rescaling). The result now follows by restricting to the interval $[0,1]$ and using two well-known facts about Sobolev spaces: that the Sobolev space $H^{n}([0,1])$ embeds continuously into $C^{k}([0,1])$ for $k<n$ and that using only the norms of the highest derivative and the function itself already gives an equivalent norm for the Sobolev space in question.

Proof of Lemma 2.3 First, we are going to prove that for all $n \in \mathbb{N}$ there exists $C_{n}>0$ such that $\left\|f^{(n)}\right\|_{L^{2}(\mathbb{R})} \leq C_{n}\|f\|_{L^{2}(\mathbb{R})}$. Indeed, we have

$$
\left\|f^{(n)}\right\|_{L^{2}(\mathbb{R})}=\left\|(2 \pi i t)^{n} \hat{f}(t)\right\|_{L^{2}(\mathbb{R})} \leq \pi^{n}\|\hat{f}(t)\|_{L^{2}(\mathbb{R})}=\pi^{n}\|f\|_{L^{2}(\mathbb{R})},
$$

where in the second step we used that supp $\hat{f} \subset\left[-\frac{1}{2}, \frac{1}{2}\right]$. 
Combining this bound with (4.1) and applying them to the half-line $[x,+\infty)$ (clearly Lemma 4.1 applies to any half-line in place of $[0,+\infty)$ ) we get

$$
\begin{aligned}
& \left|f^{(k)}(x)\right| \leq C_{k, n}\|f\|_{L^{2}([x,+\infty))}^{\alpha_{k, n}}\left\|f^{(n)}\right\| \|_{L^{2}([x,+\infty))}^{1-\alpha_{k, n}} \\
& \leq C_{k, n} C_{n}^{1-\alpha_{k, n}}\|f\|_{L^{2}([x,+\infty))}^{\alpha_{k, n}}\|f\|_{L^{2}(\mathbb{R})}^{1-\alpha_{k, n}} .
\end{aligned}
$$

Choosing $n=10 k+10$ the result now follows by noting that $\|f\|_{L^{2}([x,+\infty))} \leq$ $\|f\|_{L^{2}(\mathbb{R})}$ and $\alpha_{k, n} \geq \frac{1}{2}$ for our choice of $n$.

Acknowledgements I would like to thank Kristian Seip for sharing his hypothesis about the potential connection between Slepian's 4WT Theorem and Fourier interpolation formulas. I also would like to thank Andrii Bondarenko and Danylo Radchenko for fruitful discussions. I thank the anonymous referees for their helpful comments.

Funding Open access funding provided by NTNU Norwegian University of Science and Technology (incl St. Olavs Hospital - Trondheim University Hospital).

Open Access This article is licensed under a Creative Commons Attribution 4.0 International License, which permits use, sharing, adaptation, distribution and reproduction in any medium or format, as long as you give appropriate credit to the original author(s) and the source, provide a link to the Creative Commons licence, and indicate if changes were made. The images or other third party material in this article are included in the article's Creative Commons licence, unless indicated otherwise in a credit line to the material. If material is not included in the article's Creative Commons licence and your intended use is not permitted by statutory regulation or exceeds the permitted use, you will need to obtain permission directly from the copyright holder. To view a copy of this licence, visit http://creativecommons.org/licenses/by/4.0/.

\section{References}

1. Ascensi, G., Lyubarskii, Y., Seip, K.: Phase space distribution of Gabor expansions. Appl. Comput. Harmon. Anal. 26, 277-282 (2009)

2. Bondarenko, A., Radchenko, D., Seip, K.: Fourier interpolation with zeros of zeta and $L$-functions. arXiv:2005.02996

3. Israel, A.: The eigenvalue distribution of time-frequency localization operators. arXiv:1502.04404

4. Karnik, S., Romberg, J., Davenport, M.A.: Improved bounds for the eigenvalues of prolate spheroidal wave functions and discrete prolate spheroidal sequences. arXiv:2006.00427

5. Landau, H.J., Pollak, H.O.: Prolate spheroidal wave functions, Fourier analysis and uncertainty. III. The dimension of the space of essentially time- and band-limited signals. Bell Syst. Tech. J. 41, 1295-1336 (1962)

6. Landau, H.J., Widom, H.: Eigenvalue distribution of time and frequency limiting. J. Math. Anal. Appl. 77, 469-481 (1980)

7. Radchenko, D., Viazovska, M.: Fourier interpolation on the real line. Publ. Math. Inst. Hautes Études Sci. 129, 51-81 (2019)

8. Seip, K.: Density theorems for sampling and interpolation in the Bargmann-Fock space. I. J. Reine Angew. Math. 429, 91-106 (1992)

9. Slepian, D.: Some asymptotic expansions for prolate spheroidal wave functions. J. Math. Phys. 44, 99-140 (1965)

10. Slepian, D.: On bandwidth. Proc. IEEE 64, 292-300 (1976)

11. Slepian, D., Pollak, H.O.: Prolate spheroidal wave functions, Fourier analysis and uncertainty. I. Bell System Tech. J. 40, 43-63 (1961)

Publisher's Note Springer Nature remains neutral with regard to jurisdictional claims in published maps and institutional affiliations. 